REFERENCES:

[1] Mariette X, Criswell LA. Primary Sjogren's Syndrome. N Engl J Med. 2018;379(1):97.

[2] Catalan-Dibene J, Vazquez MI, Luu VP, Nuccio SP, Karimzadeh A, Kastenschmidt JM, et al. Identification of IL-40, a Novel B Cell-Associated Cytokine. J Immunol. 2017;199(9):3326-35.

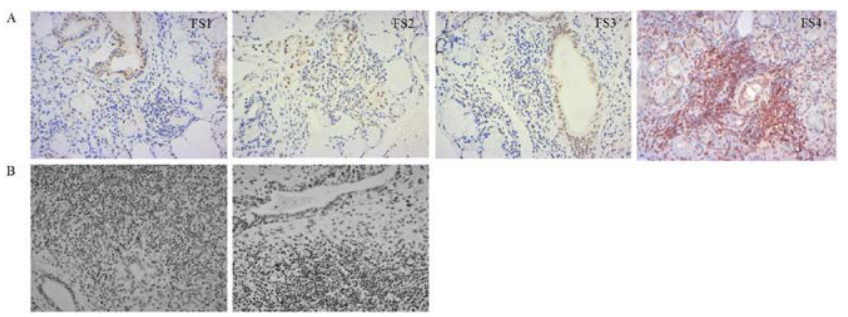

Figure 1. IL-40 overexpression in pSS and pSS-associated lymphoma A, Salivary gland biopsies stained for IL-40 showing a marked increase in IL-40 expression in presence of higher focus score. B, Parotid gland specimens of pSS-associated lymphoma showing intense staining for IL-40

Disclosure of Interests: Chiara Rizzo: None declared, Marianna Lo Pizzo: None declared, Leila Mohammadnezhad: None declared, Vincenzo Luca Lentini: None declared, Diana Di Liberto: None declared, Giulia Grasso: None declared, Piero Ruscitti Consultant of: Pfizer, Novartis, Celgene, Abbvie, Roche, Lilly, Rorberto Giacomelli Consultant of: Pfizer, Novartis, Celgene, Abbvie, Roche, Lilly, francesco ciccia Consultant of: Pfizer, Novartis, Celgene, Abbvie, Roche, Lilly, Giuliana Guggino Consultant of: Pfizer, Novartis, Celgene, Abbvie, Roche, Lilly DOI: 10.1136/annrheumdis-2021-eular.3792

\section{POS0178 PATIENTS IN SUBGROUPS WITH COMPARABLE BIOLOGICAL DISEASE PROFILES: A MULTICENTRE LONGITUDINAL STUDY}

M. J. Wahadat ${ }^{1,2}$, C. G. Van Helden-Meeuwsen ${ }^{1}$, S. Van Tilburg ${ }^{1}$, E. Schatorjé e,4 $^{3, \text { E. Hoppenreijs }}{ }^{3,4}$, P. C. E. Hissink Muller ${ }^{5}$, J. M. Van den Berg $^{6}$, D. Schonenberg-Meinema ${ }^{6}$, S. Kamphuis ${ }^{2}$, M. Versnel ${ }^{1} .{ }^{1}$ Erasmus University Medical Center, Department of Immunology, Rotterdam, Netherlands; ${ }^{2}$ Sophia Children's Hospital, Erasmus University Medical Center, Department of Pediatric Rheumatology, Rotterdam, Netherlands; ${ }^{3}$ Amalia Children's Hospital, Radboudumc, Paediatric Rheumatology, Nijmegen, Netherlands; ${ }^{4}$ St. Maartenskliniek, Paediatric Rheumatology, Nijmegen, Netherlands; ${ }^{5}$ Leiden University Medical Center, Department of Paediatrics/Paediatric Rheumatology, Leiden, Netherlands; ${ }^{6}$ Emma Children's Hospital AMC, University of Amsterdam, Department of Paediatric Immunology, Rheumatology and Infectious Diseases, Amsterdam, Netherlands

Background: Even in the hands of experienced clinicians, clinical phenotyping and predicting treatment responses in Systemic Lupus Erythematosus (SLE) patients remains challenging. Therefore, the identification of biomarkers that can help physicians to divide patients into subgroups based upon aberrantly activated pathways is relevant and might guide future treatment strategies. Extensive blood transcriptional profiling has identified various gene modules that seem promising for stratification of SLE patients into subgroups (1). However, the feasibility to implement these complicated and expensive tests for use in the daily practice of routine clinical laboratories is challenging if not impossible.

Objectives: The aim of this study was to develop gene signatures that stratify patients into groups with comparable disease profiles and are feasible to perform in routine clinical laboratories.

Methods: To identify coordinated expression of a set of genes and reduce data complexity, genes from 4 previously described modules (Interferon M1.2, Interferon M5.12, Neutrophil- and Plasmablast (PB)(1)) were measured using realtime quantitative PCR expression on whole blood RNA samples. Subsequently, a principle component analysis was used to select 2-5 indicator genes, that represent a specific signature. Expression levels of these genes were measured in healthy donors $(n=42)$ and samples from two independent childhood-onset SLE cohorts $(n=51$ and $n=20)$. Scores higher than the mean +2 S.D. score of healthy controls were defined as high gene signature scores. Based on their expression levels, gene signatures were divided over 14 clusters. Associated clusters were subsequently grouped into three gene fingerprints termed 1) all-signatures-low,
2) only high IFN (M1.2 and/or M5.12) and 3) high PB and/or Neutrophil. Disease activity was measured by the SELENA-SLEDAI score.

Results: All four gene signatures were higher expressed in patients compared to healthy controls and showed a significant correlation with the SLE DAI. The PB signature showed the highest association with disease activity ( $r=0.6512, P<0.0001)$. In longitudinally collected samples, the PB signature was reduced in patients who were on treatment and showed a significant trend with the SLEDAI. When patients were divided into the described gene fingerprints, the highest SLEDAI scores (median score $=8$ ) were observed in the high PB-Neutrophil group. The lowest disease activity (median score =2) was observed in the all-signatures-low group. The same distribution was seen when samples from a second time point were divided based on this stratification method and this was also reproduced in samples from an independent SLE cohort (figure 1).

Conclusion: Various gene signatures are associated with disease activity, which underlines the involvement of different pathophysiological mechanisms in SLE. Combining these signatures into gene fingerprints can help to stratify patients into comparable groups and guide individualized treatment choices for patients in the future.

\section{REFERENCES:}

[1] Banchereau R, Hong S, Cantarel B, Baldwin N, Baisch J, Edens M, et al Personalized Immunomonitoring Uncovers Molecular Networks that Stratify Lupus Patients. Cell. 2016;165(6):1548-50.

\section{SELENA-SLEDAI based on fingerprint distribution}

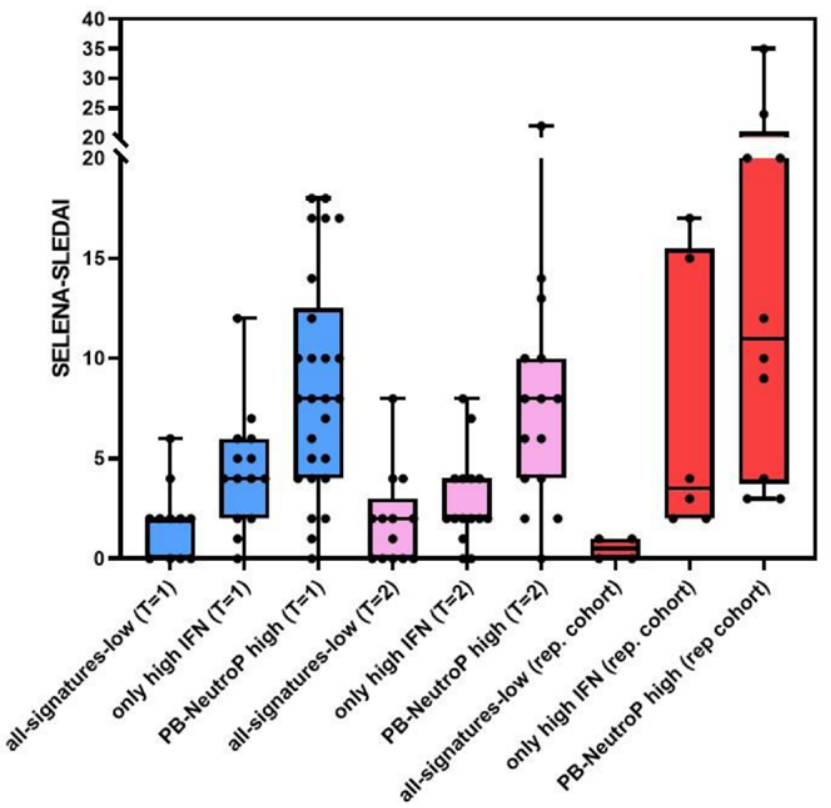

Figure 1. SELENA-SLEDAI scores based on gene fingerprint distribution. Blue indicates time point one of cohort $1(n=51)$; pink indicates time point two of cohort $1(n=45)$; red indicates time point one of the replication cohort $(n=20)$

Disclosure of Interests: None declared

DOI: 10.1136/annrheumdis-2021-eular.4011

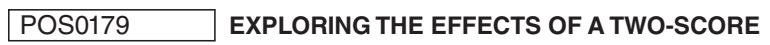 INTERFERON SIGNATURE AND RESPONSES TO INTERFERON STIMULUS}

Z. Wigston ${ }^{1}$, A. Burska ${ }^{1}$, M. Wittmann ${ }^{1}$, E. Vital ${ }^{1,2} .{ }^{1}$ University of Leeds, Leeds Institute of Rheumatic and Musculoskeletal Medicine, Leeds, United Kingdom; ${ }^{2}$ NIHR, Leeds Biomedical Research Centre, Leeds, United Kingdom

Background: IFN Scores are increasingly important as biomarkers to stratify RMDs and select patients for interferon targeted therapies. Several studies have previously demonstrated that different sets of interferon stimulated genes (ISGs) have different clinical associations. We validated a 2 -score system of ISGs expression consisting of Score A (a set of ISGs included in most interferon signature assays) and Score B (a set of less commonly 